\title{
Convergence of virulence and multidrug resistance in a single plasmid vector in multidrug-resistant Klebsiella pneumoniae ST15
}

Margaret M. C. Lam ${ }^{1}$, Kelly L. Wyres ${ }^{1}$, Ryan R. Wick ${ }^{1}$, Louise M. Judd ${ }^{1}$, Aasmund

Fostervold $^{2,3}$, Kathryn E. Holt ${ }^{1,4, *}$ Iren Høyland Löhr ${ }^{2, * \#}$

1. Department of Biochemistry and Molecular Biology, Bio21 Molecular Science and Biotechnology Institute, University of Melbourne, Parkville, Victoria, Australia

2. Department of Medical Microbiology, Stavanger University Hospital, Stavanger, Norway

3. Department of Clinical Science, University of Bergen, Bergen, Norway

4. London School of Hygiene and Tropical Medicine, London, United Kingdom

* These authors contributed equally to this work

\# Corresponding author: iren.hoyland.lohr@ sus.no 


\section{SYNOPSIS:}

Background: Multidrug resistance (MDR) and hypervirulence (hv) are typically observed in separate Klebsiella pneumoniae populations. However, convergent strains with both properties have been documented and potentially pose a high risk to public health in the form of invasive infections with limited treatment options.

Objectives: To characterize the genetic determinants of virulence and antimicrobial resistance (AMR) in two ESBL-producing $K$. pneumoniae isolates belonging to the international MDR clone ST15.

Methods: The complete genome sequences of both isolates, including their plasmids, were resolved using Illumina and Oxford Nanopore sequencing.

Results: Both isolates carried large mosaic plasmids in which AMR and virulence loci have converged within the same vector. These closely related mosaic hv-MDR plasmids include sequences typical of the K. pneumoniae virulence plasmid 1 (KpVP1 ; including aerobactin synthesis locus $i u c$ ) fused with sequences typical of $\operatorname{IncFII}_{K}$ conjugative AMR plasmids. One hv-MDR plasmid carried three MDR elements encoding the ESBL gene $b l a_{\mathrm{CTX}-\mathrm{M}-15}$ and eight other AMR genes (bla $a_{\mathrm{TEM}}$, aac3'-IIa, aph3'-Ia, dfrA1, satA2, bla $a_{\mathrm{SHV}}$, sull, aadA1). The other carried remnants of these elements encoding $b l a_{\text {TEM }}$ and $a a c 3$ '-IIa, and $b l a_{\text {CTX-M-15 }}$ was located in a second plasmid in this isolate. The two isolates originated from patients hospitalized in Norway but have epidemiological and genomic links to Romania.

Conclusions: The presence of both virulence and AMR determinants on a single vector enables simultaneous transfer in a single event and potentially rapid emergence of hv-MDR K. pneumoniae clones. This highlights the importance of monitoring for such convergence events with stringent genomic surveillance. 


\section{INTRODUCTION}

The majority of infections caused by Klebsiella pneumoniae (Kp) are typically associated with one of two distinct clinical phenomena caused by non-overlapping $K p$ populations: healthcare-associated infections caused by MDR $K p$ strains that also often cause nosocomial outbreaks, and community-acquired, invasive infections caused by hypervirulent (hv) strains ${ }^{1,2}$. However, convergent strains carrying both MDR and hypervirulent genes have been reported ${ }^{3-7}$. Recently, a high-mortality outbreak of ventilator-associated pneumonia caused by a strain of hv carbapenemaseproducing sequence type (ST) $11 K p$ was reported in China, demonstrating that the combination of enhanced virulence potential and difficulties in treatment posed by MDR can be fatal. The Chinese report was particularly notable as ST11 is typically associated with MDR, and appears to be the most common cause of carbapenemaseproducing $K p$ infections reported in China. However, the outbreak strains had additionally acquired a virulence plasmid harbouring iuc (aerobactin siderophore) and rmpA2 (hypermucoidy) loci, which are usually only observed in hypervirulent clones, such as $\mathrm{ST} 23^{2,8,9}$.

Given that antimicrobial resistance (AMR) and virulence determinants are commonly mobilized on plasmids, their occasional convergence within individual strains is not unexpected. The highly mosaic nature of $K p$ plasmids creates the risk of AMR and virulence determinants converging within a single plasmid. This hv-AMR vector could spread amongst $K p$ and confer widespread ability to cause serious infections with very limited treatment options. To our knowledge only two such plasmids have been reported; pKpvST147L, harbouring iuc, rmpA, rmpA2 and several AMR determinants (sul2, armA, sul1 and $m p h A)$ in a ST147 carriage isolate also carrying a bla $_{\mathrm{NDM}-1}$ carbapenemase and isolated in London ${ }^{4}$; and pKP70-2, harbouring the typical KpVP1 virulence plasmid of ST23 (encoding iuc, iro, rmpA and rmpA2) with an additional insertion of a MDR transposon including $b l a_{\mathrm{KPC}-2}$ carbapenemase in a K1 ST23 sputum isolate isolated in China ${ }^{10}$.

Here we report the complete genome sequences of two Kp ST15 carrying both MDR and virulence determinants, identified during a study of ESBL-producing $K p$ isolates from Norwegian hospitals. 


\section{MATERIALS AND METHODS}

\section{Ethics statement}

The isolates presented here were collected and sequenced as part of a larger national study of $K p$ in Norwegian hospitals between 2001 and 2015 called NOR-KLEB. Ethical approval for NOR-KLEB, including the collection and sequencing of $K p$ isolates and collection of patient data, was provided by the Regional ethics committee: REC west, application ID:2017/1185.

\section{Bacterial isolates}

Isolate KP_NORM_BLD_2014_104014 (KP_104014) was cultured from an 86-yearold Romanian male admitted to an Oslo hospital in 2014 with cholangiocarcinoma before developing bacteremia. Isolate KP_NORM_BLD_2015_112126 (KP_112126) was cultured from a 76-year-old female admitted to a Western Norway hospital in 2015 to treat a glioblastoma, and developed neutropenic fever with pneumonia and bacteremia. She had been hospitalized in Romania prior to admission in Norway. Antimicrobial susceptibility was determined by disk diffusion and broth micro dilution, and hypermucoidy was assessed via the string test.

\section{Whole genome sequencing and analysis}

250 and 150 bp paired end reads were generated for n=12 ST15 Norwegian $K$. pneumoniae isolates on the Illumina MiSeq and HiSeq platforms, respectively, and assembled with Unicycler v0.4.4-beta. In order to resolve the complete plasmid sequences for strains KP_104014 and KP_112126, additional long read sequencing on a MinION R9.4 flow cell (Oxford Nanopore Technologies) was performed, and combined with the Illumina short reads to generate hybrid assemblies, using Unicycler as previously described ${ }^{11,12}$, which were annotated using Prokka v1.11 ${ }^{13}$. Genotyping information including multi-locus sequence type (MLST), capsule type, AMR and virulence gene detection was extracted using Kleborate (https://github.com/katholt/Kleborate) and used to curate the annotation of relevant loci in the plasmids.

To place the hv-MDR strains in context, we performed comparative genomic analyses (described below) with an additional n=10 ST15 strains isolated between 2003 and 
2015 from seven hospitals across Norway as part of the NOR-KLEB study (full results to be reported elsewhere), together with publicly available Illumina data identified from papers reporting $K p$ ST15 genome sequences (genomes and references listed in Supplementary data 1). Illumina read data for $K p$ genomes collected by the EuSCAPE European survey of carbapenemase-producing Enterobacteriaceae ${ }^{20}$ were downloaded and assembled using Unicycler and genotyped using Kleborate to identify ST15 isolates, and the ST15 read sets were included in the comparative analysis.

All read sets were mapped to the genome of KP_104014 using the RedDog v1b 10.2 pipeline (https://github.com/katholt/RedDog). An alignment of chromosomal single nucleotide variants was extracted, recombinant regions were identified and filtered from the alignment using Gubbins v2.0.0 ${ }^{14}$, and the final alignment was passed to RAxML v8.1.23 ${ }^{15}$ to infer a core genome maximum-likelihood phylogeny. From the mapping data we also extracted the coverage of pKp104014_1 sequence, the coverage of the hv-MDR plasmid of KP_104014, and the presence of genes annotated in pKp104014_1 (presence defined as $\geq 95 \%$ of the length of the gene covered by $\geq$ five reads).

\section{Nucleotide Data Accessions}

Complete, annotated sequences for the two novel genomes have been deposited in FigShare (doi: 10.6084/m9.figshare.7222889) and GenBank (BioSamples SAMEA5063299 and SAMEA5063300). The accessions for the mosaic plasmids described here are TBC (pKp104014_1) and TBC (pKp112126_1).

\section{RESULTS AND DISCUSSION}

Isolate KP_104014 displayed resistance to cefotaxime, ceftazidime, ciprofloxacin, gentamicin, piperacillin-tazobactam and co-trimoxazole, and susceptibility to meropenem, colistin and tigecycline. The complete genome sequence resolved seven plasmids (Supplementary data 2), including a novel $346 \mathrm{kbp}$ mosaic hv-MDR

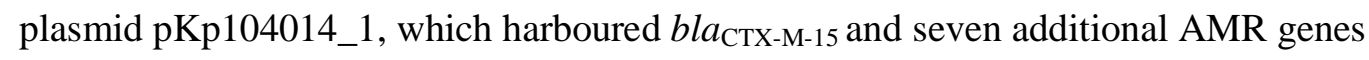
(Figure 1). Plasmid pKp104014_1 shares regions of homology with typical KpVP-1- 
type $K p$ IncFIB $_{\mathrm{K}}$ virulence plasmids ${ }^{9}$, such as pK2044 (40\% coverage, including iuc and $r m p A 2$ ), in addition to regions of homology to IncFII $_{\mathrm{K}}$ conjugative AMR plasmids (closest match: 246 kbp plasmid pKp_Goe_579-1, accession CP018313.1, from a ST147 Kp isolated in Germany, 59\% coverage). The $\mathrm{IncFII}_{\mathrm{K}}$ regions include genes for conjugative transfer, suggesting the plasmid may be self-transmissible. The plasmid harboured eight AMR genes mobilized via various elements including bla $_{\mathrm{CTX}-\mathrm{M}-15}$ (mobilized by ISEcP1); bla $a_{\mathrm{TEM}-1}$ and aac3 '-IIa (Tn3); dfrA1 and sat2

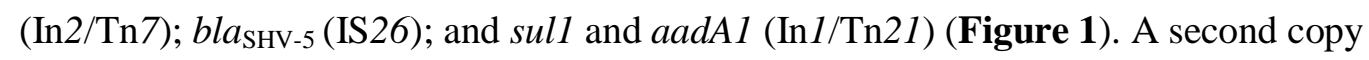
of $b l a_{\mathrm{CTX}-\mathrm{M}-15}$ was also inserted into the chromosomal gene phoE (via ISEcpl), and additional AMR genes (aacA4, bla OXA-1 $_{1}, b_{\text {TEM-1 }}$, and cat) were carried on a $76 \mathrm{kbp}$ IncFII plasmid, pKp104014_3 (Supplementary data 2).

Isolate KP_112126 displayed resistance to cefotaxime, ceftazidime, ciprofloxacin and gentamicin, intermediate susceptibility piperacillin-tazobactam and tigecycline, and susceptibility to meropenem, colistin and co-trimoxazole. The complete genome sequence resolved four plasmids, including a mosaic $299 \mathrm{kbp}$ hv-MDR plasmid pKp112126_1, with similarity to pKp104014_1 (99.99\% nucleotide identity) including $i u c, r m p A 2$, and the $\mathrm{IncFII}_{\mathrm{K}}$ transfer region. This plasmid lacks most of the AMR genes, although remnants of two AMR regions of pKp104014_1, including one end of Tn3 (encoding bla ${ }_{\mathrm{TEM}}$, aac3'-IIa) and one end of In2/Tn7 (integrase only), were present (Figure 1). Plasmid pKp112126_1 also carried an additional region with homology to KpVP1 virulence plasmids, including the ter locus encoding tellurite resistance (block L in Figure 1). bla $a_{\mathrm{CTX}-\mathrm{M}-15}$ was present in a distinct $90 \mathrm{kbp}$ plasmid, pKp112126_3, which displayed homology with pKp104014_3 and Shigella flexneri plasmid R100 (accession AP000342.1).

Both of the Norwegian hv-MDR isolates belonged to ST15 and carried the siderophore yersiniabactin (in genomic island ICEKp2) and the KL24 locus encoding capsular serotype K24. ST15 is a well-documented international ESBL-producing clone associated with nosocomial outbreaks worldwide, which frequently carries bla $_{\text {CTX-M-15-encoding IncFII plasmids }}{ }^{16-19}$. To explore the relatedness of the Norwegian isolates to one another and to the wider ST15 Kp population, we constructed a recombination-filtered, core-genome maximum likelihood phylogeny including KP_104014, KP_112126, ten additional ST15 isolates from Norway and 
306 publicly available ST15 genomes from 29 other countries (Figure 2,

Supplementary data 1). The tree showed that the two Norwegian hv-MDR isolates were closely related to one another (77 SNPs, $0.001 \%$ nucleotide divergence) and a urine isolate from Romania collected in 2013 (110 SNPs), but quite distant $(>0.003 \%$ divergent) from the other Norwegian and global isolates.

Interestingly, both the Norwegian hv-MDR plasmids were isolated from patients with epidemiological links to Romania (one of Romanian descent, one with recent travel history to Romania), suggesting the convergence of AMR and virulence plasmids may have occurred in that country rather than in Norway. The closely related Romanian isolate genome (ENA accession ERR1415588) carried iuc and rmpA2 and its reads covered $98 \%$ of the pKp112126_1 sequence and only 54\% of the typical virulence plasmid pK2044. This is consistent with the presence of a mosaic plasmid in this isolate, although the available Illumina reads were not sufficient to resolve the full sequence of the Romanian plasmid containing iuc.

bla $_{\mathrm{CTX}-\mathrm{M}-15}$ was present in most $(87 \%)$ of the ST15 genomes, along with other AMR genes (see Figure 2, Supplementary data 1). There were also multiple independent acquisitions of the ICEKp genomic island encoding yersiniabactin, affecting $48 \%$ of all ST15 isolates including 50\% of ESBL isolates (Figure 2). The only nonNorwegian ST15 isolates harbouring iuc were 30 isolates from Pakistan and the closely related Romanian isolate, all of which carried iuc and rmpA2 loci in addition to $b l a_{\mathrm{CTX}-\mathrm{M}-15}$ and multiple other AMR genes. The convergence of AMR and virulence was noted in the original study reporting these genomes from Pakistan ${ }^{21}$, however it is not possible to determine from the draft genomes whether iuc is colocalised on the same plasmid as AMR genes. Mapping of all ST15 read sets to pKp104014_1 showed that $i u c+$ isolates from Pakistan and iuc- isolates from Nepal (alongside a small number of $i u c$ - isolates from other countries) share many genes with the mosaic plasmid pKp104014_1 (55.7-68.5\% coverage for Pakistan isolates; 52.2-70.2\% coverage for Nepal isolates) (Figure 2 and Supplementary figure). This confirms that $\mathrm{IncFII}_{\mathrm{K}}$ and $\mathrm{IncFIB}_{\mathrm{K}} \mathrm{AMR}$ and virulence plasmids circulate in South Asian $K p$ ST15 populations and could potentially fuse to form hybrid hv-MDR plasmids. 
Concerningly, our findings reveal mosaic plasmids carrying both virulence determinants (iuc, rmpA2) and AMR determinants in ESBL-producing isolates of a well-established MDR $K p$ clone that has been associated with nosocomial infections and outbreaks worldwide. The co-presence of these loci in a single plasmid vector poses a substantial public health threat with the ability to simultaneously spread AMR and virulence, and it highlights the need for surveillance of virulence alongside AMR before such strains become widespread.

\section{Acknowledgments}

We thank The Norwegian Surveillance System for Antimicrobial Drug Resistance (NORM) for data sharing and The Norwegian Klebsiella pneumoniae study group for collection of Norwegian isolates.

\section{Funding}

This work was supported by The Western Norway Regional Health Authority (fellowship numbers 912037, 912119 and grant number 912050).

\section{Transparency declarations}

The authors have no conflicts of interest to disclose.

MMCL, KEH and IHL conceived the study, performed data analyses and wrote the manuscript. KLW, RRW, AF, KEH and IHL contributed additional data analysis and interpretation. AF and IHL provided isolates. LMJ performed DNA extractions and Nanopore sequencing. All authors edited and approved the manuscript. 


\section{References}

1. Shon AS, Russo TA. Hypervirulent Klebsiella pneumoniae: the next superbug? Future Microbiol 2012;7 : 669-671.

2. Holt KE, Wertheim H, Zadoks R et al. Genomic analysis of diversity, population structure, virulence, and antimicrobial resistance in Klebsiella pneumoniae, an urgent threat to public health. Proc Natl Acad Sci USA 2012; 112 : E3574-81.

3. Gu D, Dong N, Zheng Z et al. A fatal outbreak of ST11 carbapenem-resistant hypervirulent Klebsiella pneumoniae in a Chinese hospital: a molecular epidemiological study. Lancet Infect Dis 2018; 18 : 37-46.

4. Turton JF, Payne Z, Coward A et al. Virulence genes in isolates of Klebsiella pneumoniae from the UK during 2016, including among carbapenemase genepositive hypervirulent K1-ST23 and 'non-hypervirulent' types ST147, ST15 and ST383. J Med Microbiol 2018; 67 : 118-128.

5. Chen L, Kreiswirth BN. Convergence of carbapenem-resistance and hypervirulence in Klebsiella pneumoniae. Lancet Infect Dis 2018; 18 : 2-3.

6. Yao H, Qin S, Chen S et al. Emergence of carbapenem-resistant hypervirulent Klebsiella pneumoniae. Lancet Infect Dis 2018; 18: 25.

7. Wong MHY, Shum HP, Chen JHK et al. Emergence of carbapenem-resistant hypervirulent Klebsiella pneumoniae. Lancet Infect Dis 2018; 18 : 24.

8. Shon AS, Bajwa RPS, Russo TA. Hypervirulent (hypermucoviscous) Klebsiella pneumoniae: a new and dangerous breed. Virulence 2013; 4 : 10718.

9. Lam MMC, Wyres KL, Judd LM et al. Tracking key virulence loci encoding aerobactin and salmochelin siderophore synthesis in Klebsiella pneumoniae. Genome Med. 2018; 10 : 77. doi: 10.1186/s13073-018-0587-5

10. Dong N, Lin D, Zhang R et al. Carriage of blaKPC-2 by a virulence plasmid in hypervirulent Klebsiella pneumoniae. J Antimicrob Chemother 2018; doi: 10.1093/jac/dky358.

11. Wick RR, Judd LM, Gorrie C et al. Unicycler: Resolving bacterial genome assemblies from short and long sequencing reads. PLoS Comput Biol 2017; 13 : e1005595.

12. Wick RR, Judd LM, Gorrie CL et al. Completing bacterial genome assemblies with multiplex MinION sequencing. MGen 2017; 3 :1-7. 
13. Seemann T. Prokka: Rapid prokaryotic genome annotation. Bioinformatics $2014 ; 30$ : 2068-2069.

14. Croucher NJ, Page A, Connor T et al. Rapid phylogenetic analysis of large samples of recombinant bacterial whole genome sequences using Gubbins. Nucleic Acids Res 2015; 43 : e15.

15. Stamatakis A. RAxML-VI-HPC: Maximum likelihood-based phylogenetic analyses with thousands of taxa and mixed models. Bioinformatics 2006; 22 : 2688-2690.

16. Lee MY, Ko K, Kang C et al. High prevalence of CTX-M-15-producing Klebsiella pneumoniae isolates in Asian countries: diverse clones and clonal dissemination. J Antimicrob Agents 2011; 38 :160-163.

17. Breurec S, Guessennd N, Timinouni M et al. Klebsiella pneumoniae resistant to third-generation cephalosporins in five African and two Vietnamese major towns: multiclonal population structure with two major international clonal groups, CG15 and CG258. Clin Microbiol Infect 2013; 19 : 349-355.

18. Long SW, Olsen R, Eagar T et al. Population Genomic Analysis of 1,777 Extended-Spectrum Beta-Lactamase-Producing Klebsiella pneumoniae Isolates, Houston, Texas: Unexpected Abundance of Clonal Group 307. MBio 2017; 8 : e00489-17.

19. Wyres KL, Holt KE. Klebsiella pneumoniae Population Genomics and Antimicrobial-Resistant Clones. Trend Microbiol 2016; 24 : 944-956.

20. Grundmann H, Glasner C, Albiger B et al. Occurrence of carbapenemaseproducing Klebsiella pneumoniae and Escherichia coli in the European survey of carbapenemase-producing Enterobacteriaceae (EuSCAPE): a prospective, multinational study. Lancet Infect Dis 2017; 17 : 153-163.

21. Heinz E, Ejaz H, Scott J et al. Emergence of carbapenem, beta-lactamase inhibitor and cefoxitin resistant lineages from a background of ESBLproducing Klebsiella pneumoniae and K. quasipneumoniae highlights different evolutionary mechanisms. bioRxiv 2018; doi:10.1101/28. 


\section{Figure legends:}

Figure 1. Map of novel mosaic hv-MDR plasmids, showing regions of homology with closely related AMR (pKp_Goe_579-1) and virulence (pK2044) plasmids, generated using Mauve. The location of known virulence genes (blue), as well as AMR genes and their associated mobile elements (red), are also indicated.

Figure 2. Recombination-free maximum likelihood phylogeny showing virulence and antimicrobial resistance properties of 152 ST15 isolates. Tips are coloured by the country of isolation (for $\mathrm{n} \geq 4$ genomes) or geographical region as indicated, Norwegian convergent hv-MDR strains KP_NORM_BLD_2014_104014 and KP_NORM_BLD_2015_112126 labelled as A and B respectively. Columns are as follows: (1) \% Coverage of the bla $a_{\mathrm{CTX}-\mathrm{M}-15} /$ iuc plasmid pKp104014_1, determined by read mapping; (2) presence of the aerobactin synthesis locus iuc and the hypermucoidy rmpA2 gene (coloured in black); (3) yersiniabactin ICEKp variants detected; (4) capsule K-locus (KL); followed by antimicrobial resistance determinants as labelled (coloured in black for presence).

\section{Supplementary data:}

Supplementary data 1: strain table (csv)

Supplementary data 2: genome summary of Norwegian ST15 strains completed in this study (.docx)

\section{Supplementary figure. Coverage of mosaic plasmid pKp104014 amongst}

\section{Norwegian and global ST15 genomes.}

Recombination-free, maximum likelihood phylogenetic tree (left) is as shown in Fig. 1, with tips coloured by the country of isolation (for $n \geq 4$ genomes) or geographical region as indicated. Heatmap indicates the presence (coloured; see legend in top right corner) or absence (white) of AMR genes and the KpVP-1 backbone annotated in plasmid pKp104014 in each genome, with key virulence genes labelled. 


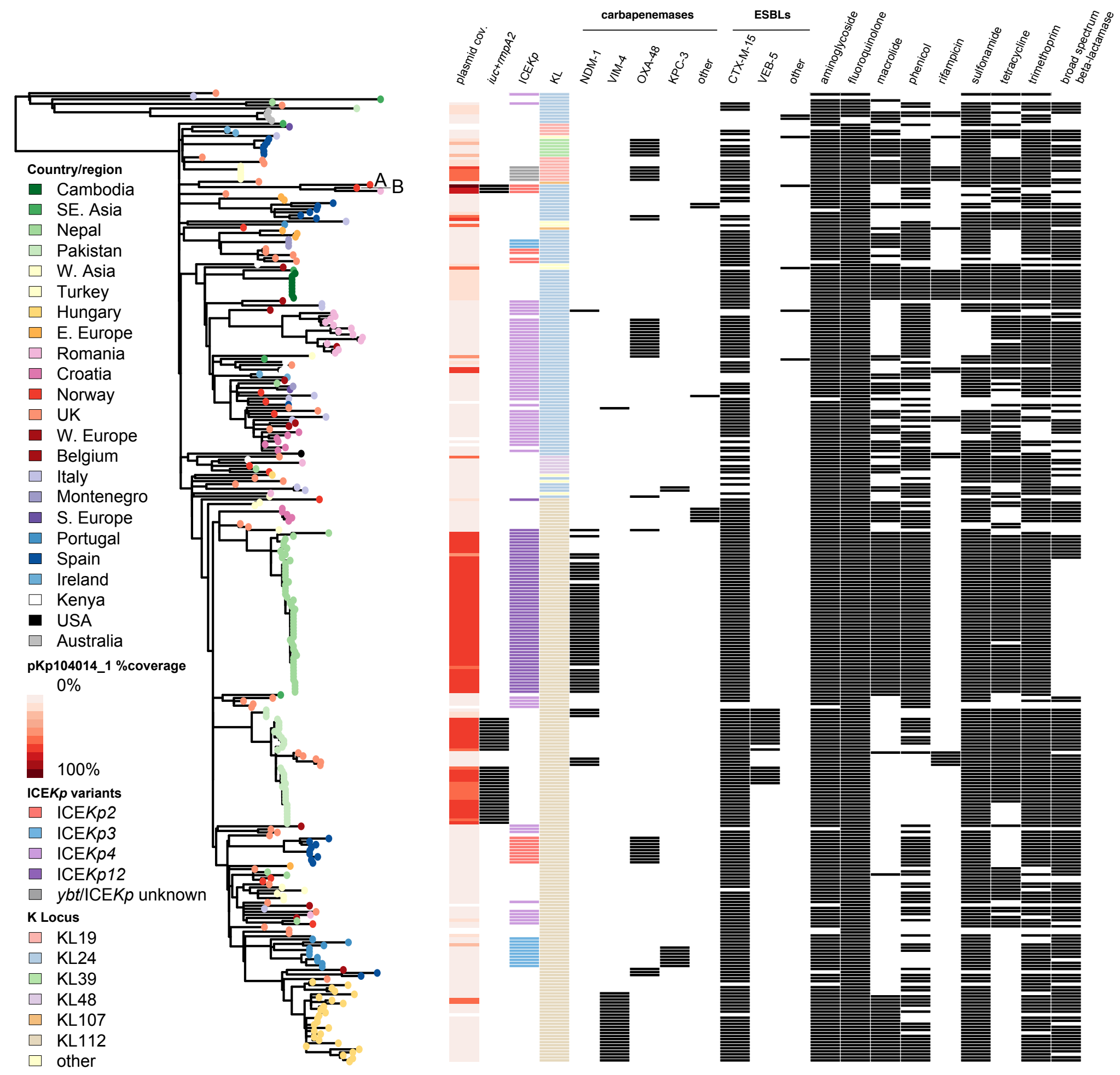

